\title{
Inter- versus intramodal integration in sensorimotor synchronization: a combined behavioral and magnetoencephalographic study
}

\author{
Katharina Müller • Gisa Aschersleben • Frank Schmitz • \\ Alfons Schnitzler · Hans-Joachim Freund • \\ Wolfgang Prinz
}

Received: 5 January 2007 / Accepted: 24 September 2007 / Published online: 12 October 2007

(C) Springer-Verlag 2007

\begin{abstract}
Although the temporal occurrence of the pacing signal is predictable in sensorimotor synchronization tasks, normal subjects perform on-the-beat-tapping to an isochronous auditory metronome with an anticipatory error. This error originates from an intermodal task, that is, subjects have to bring information from the auditory and tactile modality to coincide. The aim of the present study was to illuminate whether the synchronization error is a finding specific to an intermodal timing task and whether the underlying cortical mechanisms are modality-specific or supramodal. We collected behavioral data and cortical evoked responses by magneto-encephalography (MEG) during performance of cross- and unimodal tapping-tasks. As expected, subjects showed negative asynchrony in performing an auditorily paced tapping task. However, no asynchrony emerged during tactile pacing, neither during pacing at the opposite finger nor at the toe. Analysis of cortical signals resulted in a three dipole model best explaining tapcontingent activity in all three conditions. The temporal behavior of the sources was similar between the conditions and, thus, modality independent. The localization of the two
\end{abstract}

K. Müller $(\bowtie) \cdot$ F. Schmitz · A. Schnitzler · H.-J. Freund Department of Neurology, Heinrich-Heine University, Düsseldorf, Germany

e-mail: katharina.mueller@uni-duesseldorf.de

K. Müller · G. Aschersleben · W. Prinz

Department of Psychology,

Max Planck Institute for Human Cognitive

and Brain Sciences, Leipzig, Germany

G. Aschersleben

University of Saarland, Saarbrücken, Germany

A. Schnitzler

Wales Institute of Cognititve Neuroscience,

School of Psychology, Bangor University, Bangor, UK earlier activated sources was modality-independent as well whereas location of the third source varied with modality. In the auditory pacing condition it was localized in contralateral primary somatosensory cortex, during tactile pacing it was localized in contralateral posterior parietal cortex. In previous studies with auditory pacing the functional role of this third source was contradictory: A special temporal coupling pattern argued for involvement of the source in evaluating the temporal distance between tap and click whereas subsequent data gave no evidence for such an interpretation. Present data shed new light on this question by demonstrating differences between modalities in the localization of the third source with similar temporal behavior.

Keywords Somatosensory synchronization - MEG · Tactile stimulation $\cdot$ Somatosensory cortex

\section{Introduction}

A great amount of recent research on multisensory integration deals with the experience of perceiving synchrony of events between different sensory modalities although the signals frequently arrive at different times. Related to the sensory systems the way how multisensory integration is carried out is argued controversial (Spence et al. 2003; Sugita and Suzuki 2003; Morein-Zamir et al. 2003).

The perception of synchrony also plays a crucial role in coordinating and synchronizing motor acts to external stimulus events as required in sensorimotor synchronization tasks.

Here, participants are asked to synchronize repetitive finger-movements to extrinsic timing cues, which are mostly realized by presenting isochronous auditory stimuli. The effect typically and repeatedly found in auditory pacing is that the finger-tap precedes the tone (click) in the order to 
about 20-60 ms (e.g., Aschersleben and Prinz 1995, 1997; Dunlap 1910; Johnson 1898; Kolers and Brewster 1985; Mates et al. 1994; Miyake 1902; Thaut et al. 1998; Vos et al. 1995; for a recent review see Aschersleben 2002). Though several theoretical models have been developed to explain this synchronization error, one important aspect has widely been neglected so far. Asynchrony by mean is a phenomenon which comes about when subjects have to match two different modalities and, thus, is an intermodal effect.

The magnitude of the synchronization error has been shown to vary modality-specifically. In intermodal comparisons it has been observed that tapping to a visual pacing signal revealed smaller negative asynchrony than tapping to an auditory metronome (e.g., Bartlett and Bartlett 1959; Dunlap 1910; Miyake 1902) with mean asynchrony even becoming positive under certain conditions. To our knowledge, Kolers and Brewster (1985) presented the only fullyfledged study including an intramodal task (see Al-Attar et al. 1998 for similar results). They compared sensorimotor synchronization in three sensory modalities using auditory, visual and tactile stimuli. Tactile stimulation was realized by a $100 \mathrm{~Hz}$ vibrating mechanical stimulation via a blunted nail so that a well-defined tactile stimulus touched the left index finger. They found the magnitudes of the negative asynchrony in auditory pacing being followed by somatosensory and then visual pacing which exhibited the smallest, but at least negative, asynchrony. Another study in the context of multimodal coordination was done by Lagarde and Kelso (2006) comparing the synchronization of flexion and extension finger movements with auditory and tactile stimulation. They found the stability of multimodal coordination influenced by both the type of action and the stimulus modality and, in addition, considered the role played by time delays in multimodal coordination dynamics.

Furthermore, the duration of the pacing stimulus could play a crucial role in the arising and extent of asynchronies following Bloch's Law (Bloch 1885; see also Aschersleben 1999). Comparing, for example, electrical stimulation (which is also applied in the tactile modality; Al-Attar et al. 1998) with tactile stimulation neurophysiological data show clearly that early neuromagnetic responses evoked by electrical stimulation are different in peak amplitudes and latencies from responses evoked by tactile stimulation (e.g., Forss et al. 1994). Aiming at studying the true nature of asynchrony the attributes of the pacing stimulus should mirror the effect of the action (i.e., the tap) to the most possible extend.

There is another important aspect, that has not been taken into consideration, which is related to a theoretical approach, the so-called Paillard-Fraisse-hypothesis (Aschersleben and Prinz 1995, 1997; Fraisse 1980; Paillard 1949) or "code-generation-hypothesis". This account explains the synchronization error by differences in nerve conduction times. It is mainly based on the two assumptions that (1) the central representation of click and tap are brought to coincide and (2) the central representation of the tap is based on the somatosensory feedback from the fingertap. Therefore, the negative asynchrony is argued to be due to differences in the nerve conduction time between click and tap and their corresponding central representations. As it takes more time for the sensory information to travel from the tip of the finger to the brain than from the ear, the tap has to precede the click to establish synchronicity of the central codes. Various empirical evidence has been found in support of this hypothesis. One way to test it is to vary the effector executing the taps. As predicted by this account, comparing hand- and foot-tapping exhibited significantly increased negative asynchronies under foot- tapping conditions (Aschersleben and Prinz 1995; Billon et al. 1996; Fraisse 1980). Studies testing self-paced hand- and foot-tapping also showed a lead of the tapping foot (Bard et al. 1991, 1992; Billon et al. 1996; Paillard 1949; Stenneken et al. 2002). It is noteworthy that all these studies were comparing the effector side, i.e. the efferent part of the action. Thus, it remains unclear if and how variations of conduction times of the pacing signal (e.g., via tactile stimulation at the hand or the foot) affect asynchronies. Therefore, a first aim of the present study was to examine if the asynchrony arises during intramodal synchronization at all and if so, whether its size is determined by the position of the body part being stimulated.

A second aim was to analyze the underlying central processes in inter- and intramodal tapping tasks. To our knowledge, there is no study on this special topic whereas numerous studies have examined cortical mechanisms of sensorimotor execution and control during externally (auditorily) paced as well as self-paced finger movements (e.g., Jahanshahi et al. 1995; Müller et al. 2000; Pollok et al. 2003, 2004; Rao et al. 1993; Remy et al. 1995). What has been found were mainly differences in central timing mechanisms between external and internal pacing conditions in that during tapping without external metronom additional activation of premotor areas appeared (e.g., Boecker et al. 1994; Gerloff et al. 1998; Larsson et al. 1996; Rubia et al. 1998). The modi of temporal control, i.e. based on external versus internal timing cues, seem to be represented by different neuronal processes. Nevertheless, specific functional cortical components and, especially, neurophysiological correlates underlying the true process of precise timing and synchronization between sensory modalities are largely unknown so far.

In one of our previous studies using magnetoencephalography during an auditorily paced tapping task, direct neurophysiological correlates of different subprocesses within a synchronization sequence could be identified (Müller et al. 2000). One source was localized in contralateral primary motor cortex, the two other sources were located in 
contralateral primary somatosensory cortex. A third source was assumed to be involved in evaluating the temporal distance between tap and click. Based on the observation of a specific temporal coupling pattern which showed that both external events, tap and click, were equally triggering the central response, this source was supposed to either represent a correlate of a supramodal (i.e. higher-ranking) control function, or to be specific in crossmodal (i.e. synchronization between different modalities) synchronization, or, just as well, to be specific in matching the somatosensory effect of an action with external auditory cues. However, data of a subsequent study (Pollok et al. 2004) did not support the hypothesis of an evaluation process localized in the primary somatosensory cortex and substantiated the idea that $\mathrm{S} 1$ inferior exclusively represents the processing of somatosensory feedback information.

Comparisons of cortical sources according to a tapping task using different pacing modalities should help to clarify any higher cognitive involvement of cortical sources. Thus, sources representing modality-specific central control units should differ in aspects like temporal behavior and, above all, location. In consequence, motivation and objective of our present study was to figure out the ability to synchronize finger taps to external stimuli dependent on inter- and intramodality on the one hand, and, on the other hand, to clarify if neurophysiological correlates of achieving and controlling synchrony between uni- and crossmodal sensory and motor events are modality-specific or supramodal.

\section{Experiment}

The present study applied whole-head magnetoencephalography (MEG) to healthy human subjects in order to investigate cortical activation patterns while performing three different conditions of a sensorimotor synchronization task in subsequent runs. MEG permits noninvasive recordings of cortical activity with high temporal and spatial resolution. Compared with electric fields, magnetic fields are less distorted by intervening tissues so that the underlying neuronal sources can be located more accurately than with EEG. Behavioral data (mean asynchronies and standard deviations) were calculated and, as well as location and temporal courses of cortical sources, were compared between the different conditions.

\section{Method}

\section{Participants}

Seven healthy, right-handed individuals (4 male, 3 female), between 24 and 42 years old (mean age 29.8), were payed to participate in our study. All of them gave informed consent prior to the experiment and were musically untrained because previous studies have shown that musical training has a significant impact on mean negative asynchrony (Aschersleben 1994; Franek et al. 1991). The study was approved by the local ethics committee and is in accordance with the declaration of Helsinki. None of the participants had a history of neurological diseases.

Design

In three subsequent runs, each subject had to perform brisk flexion movements with the right index finger (1) to a binaurally presented isochronous auditory pacing-signal, (2) to a tactile isochronous pacing stimulus applied to the tip of left index finger, and (3) to a tactile isochronous pacing stimulus applied to the tip of left big toe. These three conditions were presented in a balanced order.

\section{Stimuli and apparatus}

All pacing signals were presented at an interstimulus-interval of $800 \mathrm{~ms}$. The auditory pacing signal was realized by a sine-tone at a frequency of $2,000 \mathrm{~Hz}, 82 \mathrm{~dB} \mathrm{~A}$, with a duration of $10 \mathrm{~ms}$, masked by white noise of $53 \mathrm{~dB}$ A to abolish auditory cues from external sound (e.g., tapping sound). The signals were delivered to the subject's ears through plastic-tubes to prevent any magnetic noise within the shielded room.

The tactile pacing signal was generated by a small air pressure cushion pumped up every $800 \mathrm{~ms}$ with an absolute duration from on- to offset of approximately $35 \mathrm{~ms}$. This pressure cushion was loosely but immovably fixed at either the subject's tip of left index finger or at the tip of left big toe. The perceptual impression given by this stimulus was very similar to the perceptual impression generated by the touch of the pad with the tapping finger so that subjects reported not to be able to discriminate any more between the tactile stimulus and the tactile feedback from their tap in that moment when their tap was exactly synchronous. A cardboard box hid the response apparatus and the responding finger from view, eliminating visual feedback during all runs. Tap-onset (index-finger touching the pad) was detected by a light barrier, which was fixed directly at the surface of a plastic pad. In addition, EMG-recordings were taken to control movement pattern and tap-onset-times.

\section{Procedure}

During the first 150 stimuli (about $2 \mathrm{~min}$ ) of each run, subjects merely listened to the clicks or, respectively, perceived the tactile stimuli without performing the tapping task. This procedure was necessary to record cortical 
evoked responses to the pure perception of the pacing stimuli in order to compare it to respective cortical responses during the additional performance of the synchronization task. During the next 500 stimuli subjects had to perform brisk flexion movements with the right index finger in such a way that perception of touching the pad and hearing the click or feeling the tactile stimulus, respectively, would be in exact synchrony.

\section{Recording of cortical responses}

Magnetic brain responses were measured noninvasively by a Neuromag $122^{\mathrm{TM}}$ helmet-shaped biomagnetometer. During recording, subjects were seated comfortably in a magnetically shielded room with their eyes open.

The sensor-array of the Neuromag-Magnetometer consists of 122 superconducting planar first-order gradiometers with pairs of orthogonal sensors at 61 measurement sites. Planar gradiometers detect the largest signal immediately above active cortical areas.

The MEG mainly measures current sources tangential to the skull surface; that is, activity in the cortical sulci. A head position indicator using four coils attached to the scalp delivers exact information on the position of the head relative to the sensor array before the measurement. The coil positions in relation to external anatomical landmarks (left and right preauricular points and nasion) were determined with a 3-D digitizer (Isotrak 3S1002; Polhemus Navigator Sciences, Colchester, VT) allowing realignment of MEG data and structural magnetic resonance images in the same coordinate system. Brain signals were recorded with an analog bandpass filter of $0.03-330 \mathrm{~Hz}$ and digitized at $1011 \mathrm{~Hz}$. About 300 trials were averaged with respect to the clicks and tap onsets detected by a light barrier mounted on a pad.

\section{Data analysis}

Focal cortical activation can be accounted for reasonably well by an equivalent current dipole calculated by a leastsquare estimation from the measured data at any given point of time. Spatiotemporal multidipole modeling was employed to explain the measured field patterns. Sequential dipole fitting was obtained during the whole period between two trigger points when there was a clearly dipolar field pattern. Only dipoles explaining more than $85 \%$ of the local field variance (goodness of fit) were accepted. The resulting sources were introduced into a time-varying multidipole model in which location and orientation were held constant, whereas the strength of the dipolar sources could vary.

For each subject, a structural magnetic resonance image (MRI) was generated on a $1.5 \mathrm{~T}$ Siemens-Magnetom ${ }^{\mathrm{TM}}$ (T1-weighted sequence, 128-180 sagittal slices of 1.2 to
$1.0 \mathrm{~mm}$ thickness). A spherically symmetric conductor model best fitting to the brain surface at the sensorimotor areas was obtained from the individual MRI scans and used for source localization.

\section{Calculation of behavioral data}

Registering different timepoints during the performance of each run within the tapping task allowed the calculation of behavioral data. For each subject, differences between each trigger point (pacing onset $p$ and tap onset $t$ ) were calculated and the results were subtracted. The resulting values representing individual mean asynchrony $(t-p)$ and standard deviation were averaged across all subjects.

\section{Results}

Behavioral results

As expected, subjects produced a group mean negative asynchrony $(N=7)$ of $-39 \mathrm{~ms}$ with an interindividual standard deviation (SD) of $26 \mathrm{~ms}$ during auditory pacing. Mean intraindividual SD was $37 \mathrm{~ms}$. Tactile pacing at the left index finger exhibited a group mean negative asynchrony of $-8 \mathrm{~ms}$ with an interindividual standard deviation of $9 \mathrm{~ms}$ (mean intraindividual SD $42 \mathrm{~ms}$ ), whereas tapping during tactile pacing at the left big toe resulted in a group mean negative asynchrony of $-4 \mathrm{~ms}$ with an interindividual standard deviation of $32 \mathrm{~ms}$ (mean intraindividual SD $51 \mathrm{~ms}$ ). Table 1 shows mean asynchronies and standard deviations of all subjects during the three conditions.

The $t$ tests showed no significant difference between the value of asynchrony and zero, neither in the tactile pacing finger condition $(t=1.76 ; P>0.13)$ nor in the tactile pacing toe condition $(t=0.146 ; P>0.50)$, whereas the asynchrony during auditory pacing was significantly negative and different from zero $(t=4.45 ; P=0.004)$. Obviously, tapping during auditory pacing exhibits a significant negative asynchrony whereas tapping during tactile pacing is synchronous by means, no matter where the pacing signal is applied.

Comparing intraindividual SDs of asynchronies with Wilcoxon nonparametric test showed no statistically significant difference between intraindividual SD of tactile finger and tactile toe stimulation and also no difference between intraindividual SD of tactile finger and auditory stimulation whereas the difference between tactile toe and auditory stimulation became significant $(0.024)$. As could have been expected from previous studies stability of sensorimotor coordination is constricted with the body part being stimulated (and also being the effector; see Aschersleben 1994). 
An analysis of variance for repeated measurements comparing asynchrony in the three conditions resulted in a main effect for condition; $F(2 ; 10)=6.66 ; \quad P<0.015 ; \mathrm{ETA}^{2}=$ 0.57. Post-hoc paired comparisons between the conditions yielded no significant difference between the tactile pacing condition at the finger and at the toe $(P>0.50)$; the difference between the auditory pacing condition and the tactile pacing finger condition was significant $(P<0.02)$ as well as the difference between auditory pacing and the tactile pacing toe condition $(P<0.02)$. Thus, the difference between sensory modalities is statistically relevant whereas the position of stimulus deliverance yields no statistically significant difference in asynchronies despite a significant difference in intraindividual SD between tactile toe and auditory stimulation, which is in line with previous studies.

\section{Cortical responses}

In all three conditions, during the first 150 trials of merely sensory stimulation evoked brain responses were averaged related to the onset of the sensory pacing signal (auditory click or tactile stimulus at the finger and at the toe, respectively). In all subjects, source modeling applied to auditory evoked responses detected between two and four sources bilaterally in supratemporal gyrus with maximal amplitudes around 50 and $100 \mathrm{~ms}$. These sources most probably represent activation of primary and associative auditory cortices.

In all subjects tactile evoked fields to pacing stimuli at the left index finger were explained by two to three sources contralaterally in primary somatosensory cortex (peaking around $40 \mathrm{~ms}$ ) and secondary somatosensory cortex (peaking around $60 \mathrm{~ms}$ ). A one to two dipole-model explained tactile evoked fields to pacing stimuli at left big toe. These sources were located contralaterally in primary somatosensory cortex (peaking around 50-70 ms) and, if identifiable, in second somatosensory cortex (peaking around 60-80 ms after stimulus onset).

During the whole tapping sequence, evoked responses were averaged in two ways, time-locked to the onset of the sensory pacing signal and time-locked to tap-onset (indexfinger touching the pad). With auditory metronome, the brain responses averaged time-locked to tap-onset showed maximal amplitudes over the contralateral rolandic area. The tap-related magnetic fields during tactile pacing at the left index finger and at left big toe looked quite similar. Figure 1 shows an overlay of the averaged responses for all three conditions time-locked to the tap in one representative subject. Here, evoked responses still contain the contributions of the pacing signals in the overall field distributions.

In a next step, the portion of the field distribution explained by the sources activated by mere sensory stimulation without tapping (as described above) in each condition was weighted and subtracted from the tap-related field distribution using the signal-space-projection method (e.g., Tesche et al. 1995). In all three conditions, the remaining field distributions could be best explained by a three dipole model in six of the seven subjects. In one subject, only two sources could be detected to explain the field distributions. Location and temporal courses of these two sources were comparable with the two later peaking sources detected in the other subjects. In the three dipole-model, the first dipolar source showed its maximum approximately $100 \mathrm{~ms}$ before tap-onset (this source could not be detected in one of the subjects), a second source was mainly active approximately
Fig. 1 Magnetic evoked responses averaged to tap onset in one representative subject for the three conditions "tapping to auditory pacing" (grey curves), "tapping to tactile pacing at left index finger" (black curves), and "tapping to tactile pacing at left big toe" (dashed curves). Responses are depicted from $200 \mathrm{~ms}$ before to $200 \mathrm{~ms}$ after tap onset. Zooms on the left-side show activity over the contralateral rolandic area and posteriorparietal area. Magnetic field gradients were measured along latitudes and longitudes, as illustrated by the schematic heads in the upper right corner

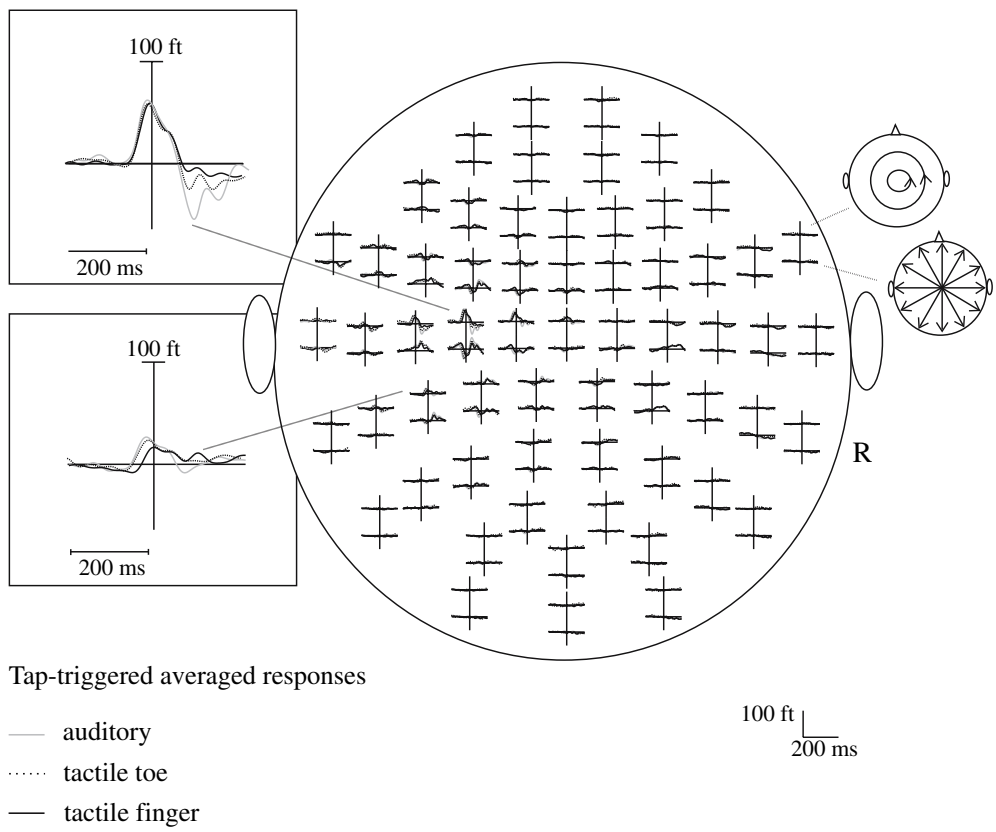


at tap-onset $(0 \mathrm{~ms})$, and the third source showed peak amplitude around $80 \mathrm{~ms}$ after tap-onset. Periods and order of peaking of the sources were comparable between the three conditions. Figure 2 illustrates the time courses of the three sources comparing the three conditions "tapping during auditory pacing", "tapping during tactile pacing at left index finger", and "tapping during tactile pacing at left big toe" by grandaverages for all seven subjects (the first dipole contains the data of only six subjects).

Analysis of source localizations yielded an interesting difference: In all three conditions, the first tap-related source was localized in contralateral primary motor cortex (M1), probably reflecting executive motor activity, and the second source was located in contralateral primary somatosensory cortex (S1), most probably representing afferent and reafferent feedback. On the contrary, a third tap-related source which - as outlined above-showed no difference in

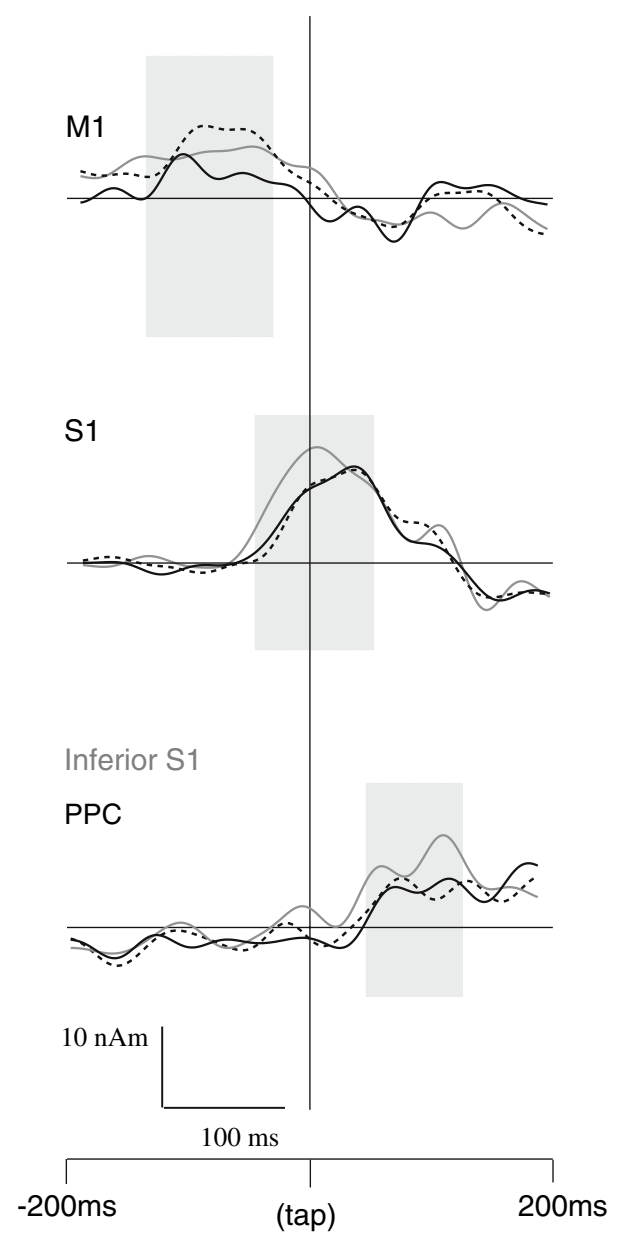

Fig. 2 Activity of tap-related sources (grandaverage, $N=7$ ) as a function of time. Tap-related source waveforms of the conditions "tapping to auditory pacing" (grey line), "tapping to tactile pacing at left index finger" (black line), and "tapping to tactile pacing at left big toe" (dashed line) are plotted on top of each other. The time windows of individual peak amplitudes are highlighted in light grey temporal behavior between the conditions, was also located in contralateral primary somatosensory cortex inferior to the second source during auditory pacing, but was located in contralateral posterior-parietal cortex (PPC) during tactile pacing, no matter whether the pacing signal was applied to the left index finger or to the left big toe. Thus, location of the third tap-relevant source seemed to be dependent of the modality of the pacing signal.

This pattern can already be observed by a comparison of the field distributions in contour maps at the critical point of time (Fig. 3a). In addition, the localization difference is shown by an overlay of the sources of one representative subject on his brain surface rendering (Fig. 3b).

This localization pattern could be found quite uniformly in five of the seven subjects; in one subject, the third source during tactile pacing was localized more medially but nevertheless in PPC, and in one subject, all sources were localized more anteriorly in general. Figure 4 shows, for all subjects, locations of PPC sources activated during tapping to tactile pacing in the axial and coronal plane of the MEG coordinate system relative to the inferior $\mathrm{S} 1$ source active during tapping to auditory pacing. In addition, mean difference and standard deviation are drawn in.

As is already indicated in the axial view, the PPC source is located significantly more posterior than the inferior S1 source. The localization difference on the $y$-axis is significantly different (Wilcoxon test, $P=0.043$ ). The coronal view indicates that the difference on the $z$-axis (superior to inferior) is not statistically significant $(P>0.20)$, although there is a tendency of the posterior-parietal sources to be located more inferior (compared to inferior S1). Mean $95 \%$ confidence limits for dipole localization are $5.72 \mathrm{~mm}^{3}$ for the inferior $\mathrm{S} 1$ sources and $6.12 \mathrm{~mm}^{3}$ for the PPC sources.

\section{Discussion}

The aim of the present study was to investigate the influence of the modality of the pacing signal on the timing of the motor response as well as on the underlying cortical mechanisms. In the following, we first will discuss the behavioral results and, second, the central data.

\section{Behavioral results}

Behavioral results replicate common findings of previous studies in that during auditory pacing subjects exhibit a mean negative asynchrony of approximately $40 \mathrm{~ms}$. In contrast to the rare previous studies which had found smaller but still negative asynchronies under somatosensory stimulation (Al-Attar et al. 1998; Kolers and Brewster 1985) no significant asynchrony emerged in our study when fingertaps were synchronized to a tactile metronome, neither 


\section{A Auditory pacing}
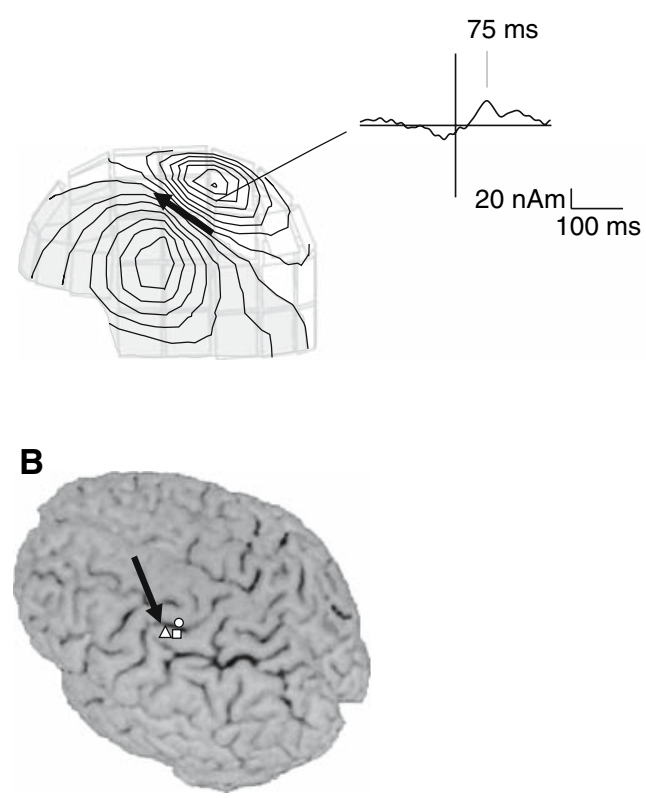

M1

$\square \mathrm{S} 1$

$\triangle \mathrm{S} 1$ inferior

Fig. 3 a Magnetic field patterns of tap-related responses at approximately $75 \mathrm{~ms}$ after tap-onset and corresponding current sources (arrows) and source waveforms for the auditory pacing condition (left) and the tactile finger pacing condition (right) in one representative subject. The helmet-shaped sensor array is viewed from the left. Arrows represent location and direction of dipoles, corresponding source strengths as a function of time are shown on the right of the sensor

during tactile pacing at the left index finger nor during tactile pacing at the left big toe.

This finding is interesting in various respects. First of all, the different results in the literature can be explained by the use of different kinds of tactile stimuli. Kolers and Brewster (1985) used a short and well defined tactile stimulus touching the left index finger, Al-Attar et al. (1998) applied an electrical stimulation whereas the present study used a small air pressure cushion, which is much more similar to the stimulation of the finger when touching the response pad. Moreover, neurophysiological data clearly show that early neuromagnetic responses evoked by electrical stimulation are larger in peak amplitudes and shorter in latencies compared to responses evoked by tactile stimulation (e.g., Forss et al. 1994).

Second, in accordance with what the code-generationhypothesis would predict, the negative asynchrony disappeared when nerve conduction time was the same for the sensory information of the pacing signal and the sensory feedback generated by touching the pad with the index finger. However, the code-generation-hypothesis would
Tactile pacing
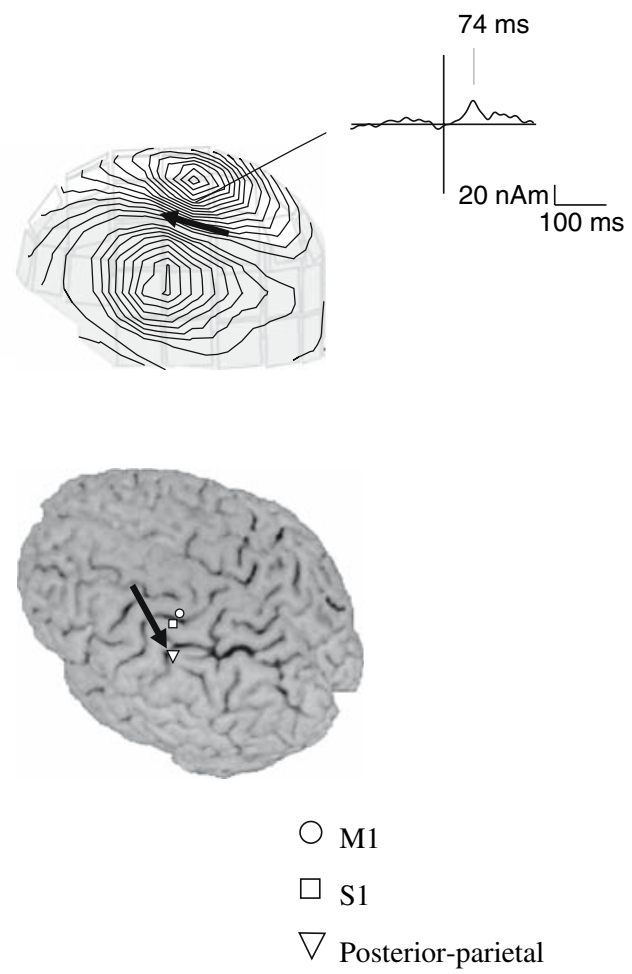

arrays. b The three tap-related sources superposed on the brain surface rendering of one representative subject. During auditory pacing (left) and tactile pacing (right) the same areas in M1 (circle) and S1 (rectangle) are activated, whereas the ,third" source (triangle) is located in inferior S1 cortex (triangle) during auditory pacing and in posterior parietal cortex (upside-down triangle) during tactile pacing

also assume that nerve conduction times of the sensory signals should be different in the finger- and the toe-condition resulting in different asynchronies for the two tactile pacing conditions (the toe-condition should reveal positive asynchronies). This was definitely not the case in our study. In consequence, the finding of our experiment cannot be sufficiently explained by the assumptions of the code-generation-hypothesis. An important difference to previous experiments is the fact that our study is looking at the afferent path whereas former studies had focused on the efferent path. Rather, our results suggest that subjects are able to take into account differences in nerve conduction time at least on the afferent path and if the pacing signal is presented in the same modality as the event to be synchronized. Thus, the synchronization error seem to be a finding specific to an intermodal timing task.

\section{Central data}

Analysis of cortical evoked responses resulted in three sources mainly explaining tap-related activity in all three 

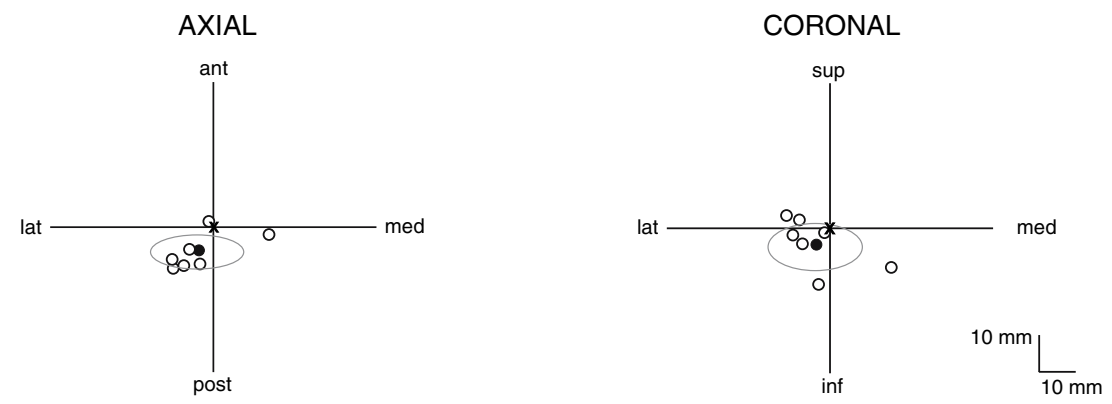

X coordinate centre $=\mathrm{SI}$ inferior source (auditory pacing)

- posterior-parietal source (tactile pacing)

standard deviation of source localization differences

- mean localization of posterior-parietal source

Fig. 4 Locations of PPC sources (tactile pacing) with respect to inferior S1 sources (auditory pacing) in all subjects plotted on the axial (left) and coronal plane (right) of the MEG coordinate system. The black cross shows the overlay of individual S1 sources as coordinate centre; the black-edged circles represent the PPC-sources referenced to

conditions. During auditory pacing the first of these sources had its peak amplitude approximately at $100 \mathrm{~ms}$ before taponset and is located in M1, a second source peaks around tap-onset and was localized in S1, and the third source which showed localization in $\mathrm{S} 1$ inferior to the earlier S1 source had the peak amplitude around $80 \mathrm{~ms}$ after taponset. Thus, these data replicated the finding of earlier studies which yielded similar activation pattern (Müller et al. 2000; Pollok et al. 2004). In the tactile pacing conditions (both, at the left index finger and at the left big toe), temporal courses of the three sources were comparable in latencies of their peak amplitudes. But, whereas in the tactile pacing conditions the two earlier sources were located in the same areas as during tapping under auditory pacing, the third source was located in PPC with a tendency to be more inferior than the second S1 source of the auditory pacing condition.

This result opens an interesting sight on the background of previous neurophysiological data in the context of sensorimotor synchronization: In an earlier study where subjects only had to synchronize to an auditory pacing signal the third source which was located in S1 inferior was analyzed due to its time locking to the external events "tap" and "click". In these data, this source was equally well time locked to "tap" and "click", and, beyond that, the respectively second trigger event (tap or click) depending on the direction of the asynchrony, was crucial for the source to be activated (Müller et al. 2000). Hence, the data gave reason to suppose this third source being involved in evaluation of the distance between "tap" and "click". However, although a subsequent study could replicate the finding of the same three mainly active sources, the temporal coupling pattern the coordinate centre; the grey-edged circle shows the standard deviation of source localization differences, and the black-filled circle represents the mean localization of PPC-source. Note that the scaling on the right applies to all diagrams. ant anterior; post posterior; lat lateral; med medial; sup superior; inf inferior

could not be shown (Pollok et al. 2004). A possible explanation for the different findings might be that the distribution pattern of the peripheral (asynchronies) data and the corresponding central responses were different in the way that the relation of peripheral and central jitter (the variability in the peripheral click-tap intervals and the variability in central responses) changed. As our data are not productive with respect to the examination of distribution pattern, further studies with aimful variations of the click-tap-distance, e.g. by instruction could help to further clarify this question.

In the present study, the localization of the third source was modality-specific, that is, it was located more inferior under tactile pacing conditions than under auditory pacing conditions. The corresponding behavioral data showed no synchronization error during tactile pacing whereas during auditory pacing subjects repeatedly reveal negative asynchronies. Previous neurophysiological data on sensory stimulation within one single modality indicated that nerve conduction times do not differ significantly between the auditory and the somatosensory modality. Earliest cortical responses in both modalities are found at approximately 30-50 ms after stimulation (e.g., Büttner 1996; Claus et al. 1987; Forss et al. 1994). Different nerve conduction times for different sensory systems are mainly important on brainstem level. The argumentation of code-generation-hypothesis does not rely on a specific brain level; in the original version it referred to pure peripheral levels. Thus, nerve conduction time probably plays a role in sensorimotor synchronization but not on the cortical level.

Comparing the subprocesses possibly involved in the two types of synchronization tasks, the intermodal task and 
the intramodal one, the evaluation and control mechanisms should be distinguished in processes relying more on temporal characteristics and those relying more on spatial criteria. Whereas in the intermodal task the criterion of spatial separation between pacing and action effect is of no importance (because of two different modalities being involved), the temporal judgement-i.e. being early or being late-is well to the fore. Evidence for this assumption is given by our previous data where the temporal relations between the decisive cortical source and the peripheral events tap and click clearly refer to a temporal evaluation mechanism. This temporal judgment is as more difficult as more it is based on different pathways of information processing that have to be integrated - in consequence, subjects show asynchronies. In case of the intramodal task, the sensory information processing is identical and, in that, precise temporal evaluation is easy-subjects do not perform asynchronies. But, at the same time, the two sources of information on which their temporal judgment is based become blurred and indistinct. Indeed, our subjects uniformly reported not to be able to discriminate any more between the "perceptual" side and the "effector" side in that moment when they felt to be in exact synchrony. Thus, instead of being able to evaluate their timing on the basis of temporal information, they have to replace this noninterpretable information by another dimension, the spatial one. That is, they have to separate pacing signal and sensory feedback from the effector on the basis of their spatial distance.

Latency and localization of the crucial cortical source identified in our intramodal task gives support to these assumptions: PPC has long been considered a sensory area specialized for spatial awareness and the directing of attention (e.g., Mesulam 1999), especially in visuospatial processing and accessing sensorimotor knowledge (e.g., Sugio et al. 1999). On that background, thinking about what subjects have to do when evaluating the distance between the peripheral sensory events, "evaluation-neurons" might help to discriminate the two sensory events. The activation of PPC during evaluation could, therefore, represent some kind of spatial discrimination between "preceptor" and "effector" (to be able to evaluate their distance), only relevant during intramodal synchronization whereas in intermodal synchronization discrimination should be possible by sensory attributes (in that the modalities should differ in their attributes both, qualitatively and quantitatively).

Why, then, should in auditorily paced tapping a function like "evaluation" be localized in primary somatosensory cortex? Some but so far rare studies give a few hints to S1 possibly being involved in some higher level cognitive processes. For example, one PET-study investigated cortical activation under tactile attention by discriminating tactile features like roughness and length. The data suggested involvement of S1 in attentional processes on the one hand and, second, an increasing S1 activity under conditions when tactile information was behaviorally relevant whereas activation was depressed when information about other modalities or tasks had to be processed (Burton et al. 1999). Others refer to the participation of somatosensory neurons not only in perception but in short-term memory for tactile stimuli by applying single-cell recordings in monkeys (Zhou and Fuster 1996).

In addition, studies exploring crossmodal plasticity in cases if one specific sensory modality is missing (Cohen et al. 1997, 1999; Kujala et al. 1996; Rauschecker 1995; Roder et al. 1997; Sadato et al. 1996; Uhl et al. 1993) could demonstrate that a loss of a specific sensory modality contributes to sensory compensation. Blindfolded subjects, for example, show activation of visual areas during performance of tactile (Braille reading) or auditory tasks (e.g., Cohen et al. 1999, 1997; Sadato et al. 1996). These results give support to the assumption that processes of sensory compensation and substitution might also play a role in uniand crossmodal synchronization. Nevertheless, based on our data we are not able to determine whether the localization difference of our third source is modality specific or, on the other hand, specific for uni- (PPC) or crossmodal evaluation as we compared only one uni- with one crossmodal paradigm. This has to be clarified in further experiments.

In sum, our study yielded interesting results with respect to higher-level cognitive mechanisms being involved in sensorimotor synchronization and the crucial role of the pacing modality in precision. The functional role of the involved areas has to be clarified more specifically in further experiments.

\section{References}

Al-Attar Z, O'Boyle DJ, Cody FWJ (1998) Effects of site of delivery of an electrical cutaneous metronome on the magnitude of the synchronization error during human temporal tracking. J Physiol 509P: $181-182$

Aschersleben G (1999) Aufgabenabhängige Datierung von Ereignissen. Shaker, Aachen

Aschersleben G (2002) Temporal control of movements in sensorimotor synchronization. Brain Cogn 48:66-79

Aschersleben G, Prinz W (1995) Synchronizing actions with events: the role of sensory information. Percept Psychophys 57:305-317

Aschersleben G, Prinz W (1997) Delayed auditory feedback in synchronization. J Motor Behav 29(1):35-46

Aschersleben G, Gehrke J, Prinz W (2001) Tapping with peripheral nerve block: a role for tactile feedback in the timing of movements. Exp Brain Res 136:331-339

Bard C, Paillard J, Lajoie Y, Fleury M, Teasdale N, Forget R, Lamarre $Y$ (1992) Role of the afferent information in the timing of motor commands: a comparative study with a deafferent patient. Neuropsychologia 30:201-206

Bard C, Paillard J, Teasdale N, Fleury M, Lajoie Y (1991) Self-induced versus reactive triggering of synchronous hand and heel movement in young and old subjects. In: Requin J, Stelmach E (eds) 
Tutorials in motor neuroscience. Kluwer, Amsterdam, pp 189196

Bartlett NR, Bartlett SC (1959) Synchronization of a motor response with an anticipated sensory event. Psychol Rev 66:203-218

Billon M, Bard C, Fleury M, Blouin J, Teasdale N (1996) Simultaneity of two effectors in synchronization with a periodic external signal. Hum Mov Sci 15:25-38

Bloch AM (1885) Experiences sur la vision. Comptes endus de la Societe Biologique 37(493)

Boecker H, Kleinschmidt A, Requardt M, Hanicke W, Merboldt KD, Frahm J (1994) Functional cooperativity of human cortical motor areas during self-paced simple finger movements. A high-resolution MRI-study. Brain 117:1231-1239

Burton H, Abend NS, MacLeod A-MK, Sinclair RJ, Snyder AZ, Raichle ME (1999) Tactile attention tasks enhance activation in somatosensory regions of parietal cortex: a positron emission tomography study. Cereb Cortex 9:662-674

Büttner UW (1996) Akustisch evozierte potentiale (Auditory evoked potentials). In: Stoehr M, Dichgans J, Büttner UW, Hess ChW, Altenmüller E (eds) Evozierte potentiale. Springer, Berlin, pp 411-486

Claus D, Linsenmeier R, Sturm U, Engelhardt A (1987) Somatosensory evoked potentials following tactile skin stimulation. EEG MEG 18(4):115-121

Cohen LG, Celnik P, Pauscual-Leone A et al (1997) Functional relevance of cross-modal plasticity in the blind. Nature 74:180-183

Cohen LG, Weeks RA, Sadato N, Celnik P, Ishii K, Hallett M (1999) Period of susceptibility for cross-modal plasticity in the blind. Ann Neurol 45:451-460

Dunlap K (1910) Reactions on rhythmic stimuli, with attempt to synchronize. Psychol Rev 17:399-416

Forss N, Salmelin R, Hari R (1994) Comparison of somatosensory evoked fields to airpuff and electric stimuli. Electroencephalogr Clin Neurophysiol 92:510-517

Fraisse P (1980) Les synchronisations sensori-motrices aux rythmes [Sensorimotor synchronizations to rhythms]. In: Requin J (ed) Anticipation et comportement. Centre National, Paris, pp 233 257

Franek MM, Mates J, Radil T, Beck K, Pöppel E (1991) Finger tapping in musicians and nonmusicians. Int J Psychophysiol 11:187-192

Gerloff C, Richard J, Hadley J, Schulman AE, Honda M, Hallett M (1998) Functional coupling and regional activation of human cortical motor areas during simple, internally paced and externally paced finger movements. Brain 121:1513-1531

Jahanshahi M, Jenkins IH, Brown RG, Marsden CD, Passingham RE, Brooks DJ (1995) Self-initiated versus externally-triggered movements. I. An investigation using measurement of regional cerebral blood flow with PET and movement related potentials in normal and Parkinson's disease subjects. Brain 118:913-33

Johnson WS (1898) Researches in practice and habit. Stud Yale Psychol Lab 6:51-105

Kolers PA, Brewster JM (1985) Rhythms and responses. J Exp Psychol Hum Percept Perform 11:150-167

Kujala T, Alho K, Kekoni J et al (1995) Auditory and somatosensory event-related brain potentials in early blind humans. Exp Brain Res 104:519-526

Lagarde J, Kelso JAS (2006) Binding of movement, sound and touch: multimodal coordination dynamics. Exp Brain Res 173/4:673688

Larsson J, Gulyas B, Roland PE (1996) Cortical representation of selfpaced finger movement. Neuroreport 7(2):463-468

Mates J, Müller U, Radil T, Pöppel E (1994) Temporal integration in sensorimotor synchronization. J Cogn Neurosci 6:332-340

Mesulam MM (1999) Spatial attention and neglect: parietal, frontal and cingulate contributions to the mental representation and atten- tional targeting of salient extrapersonal events. Philos Trans $\mathrm{R}$ Soc Lond B Biol Sci 43:1325-1346

Miyake J (1902) Researches on rhythmic action. Stud Yale Psychol Lab 10:1-48

Morein-Zamir S, Soto-Faraco S, Kingstone A (2003) Auditory capture of vision: examining temporal ventriloquism. Brain Res Cogn Brain Res 17(1):154-163

Müller K, Schmitz F, Aschersleben G, Schnitzler A, Freund H-J, Prinz W (2000) Neuromagnetic correlates of sensorimotor synchronization. J Cogn Neurosci 12:1-10

Paillard J (1949) Quelques donnees psychophysiologiques relatives au declenchement de la commande motrice [Some psychophysiological data relating to the triggering of motor commands]. L'Ann. Psych 48:28-47

Pollok B, Müller K, Aschersleben G, Schmitz F, Schnitzler A, Prinz W (2003) Cortical activations associated with auditorily paced finger tapping. NeuroReport 14(2):247-250

Pollok B, Müller K, Aschersleben G, Schnitzler A, Prinz W (2004) The role of the primary somatosensory cortex in an auditorily paced finger tapping task. Exp Brain Res 156:111-117

Rao SM, Binder JR, Bandettini PA, Hammeke TA, Yetkin FZ, Jesmanowicz A, Lisk LM, Morris GL, Mueller WM, Estkowski LD et al (1993) Functional magnetic resonance imaging of complex human movements. Neurology 43:2311-2318

Rauschecker JP (1995) Compensatory plasticity and sensory substitution in the cerebrale cortex. Trends Neurosci 18:36-43

Remy P, Zilbovicius M, Leroy-Willig A, Syrota A, Samson Y (1995) Movement- and task-related activations of motor cortical areas: a positron emission tomography study. Ann Neurol 36:19-26

Röder B, Rösler F, Henninghausen E (1997) Different cortical activation patterns in blind and sighted humans during encoding and transformation of haptic images. Psychophysilogy 34:292-307

Rubia K, Overmeyer S, Taylor E, Brammer M, Williams S, Simmons A, Andrew C, Bullmore E (1998) Prefrontal involvement in "temporal bridging" and timing movement. Neuropsychologia 36(12):1283-1293

Sadato N, Pascual-Leone A, Grafman J et al (1996) Activation of the primary visual cortex by Braille reading in blind subjects. Nature 380:526-528

Spence C, Squire S (2003) Multisensory integration: maintaining the perception of synchrony. Curr Biol 13(13):R519-R521

Stenneken P, Aschersleben G, Cole J, Prinz W (2002) Self-induced versus reactive triggering of synchronous movements in a deafferented patient and control subjects. Psychol Res 66:40-49

Sugio T, Inui T, Matsuo K, Matsuzawa M, Glover GH, Nakai T (1999) The role of the posterior parietal cortex in human object recognition: a functional magnetic resonance imaging study. Neurosci Lett 276(1):45-48

Sugita Y, Suzuki Y (2003) Audiovisual perception: implicit estimation of sound-arrival time. Nature 421(6926):911

Tesche CD, Uusitalo MA, Ilmoniemi RJ, Huotilainen M, Kajola M, Salonen O (1995) Signal-space projections of MEG data characterize both distributed and well-localized neuronal sources. Electroencephalogr Clin Neurophysiol 95(3):189-200

Thaut MH, Tian B, Azimi-Sadjadi MR (1998) Rhythmic finger tapping to cosine-wave modulated metronome sequences: Evidence of subliminal entrainment. Hum Mov Sci 17:836-839

Uhl F, Franzen P, Podreka I et al (1993) Increased regional cerebral blood flow in inferior occipital cortex and cerebellum of early blind humans. Neurosci Lett 150:162-164

Vos PG, Mates J, van Kruysbergen NW (1995) The perceptual centre of a stimulus as the cue for synchronization to a metronome. Q J Exp Psychol 48:1024-1040

Zhou Y-D, Fuster JM (1996) Mnemonic neuronal activity in somatosensory cortex. Proc Natl Acad Sci USA 93:10533-10537 